\title{
An experimental investigation of ground resonance and air resonance of bearingless model rotor
}

\author{
Yan Zhu ${ }^{\text {a }}$, Yuhui Lu, Zhizhuang Feng, Quanlong Chen and Feng Qian \\ Science and Technology on Rotorcraft Aeromechanics Laboratory, CHRDI, Jingdezhen, China
}

\begin{abstract}
A stability experimental method of ground and air resonance for bearingless rotor on test-bed was investigated, the test principle and method were presented. A 4-m diameter rotor model was used to investigate aeromechanical stability of ground and air resonance and the results were compared to theory, the measured lead-lag regressing mode damping agreed well with theory. The results indicated this method has advantages of reliability and validity, improves test security, achieves the desired purposes, and provides effective technology for the bearingless rotor helicopter stability test.
\end{abstract}

Keywords: bearingless rotor, test bench, ground resonance, air resonance, test method.

\section{Introduction}

At present the bearingless rotor is the most advanced configuration of helicopter rotor system, which uses the composite material flexbeam replacing flapping hinge and lead-lag hinge and pitching hinge in conventional articulated rotor hub. The flexbeam performs the motions of flapping and lead-lag and pitching by elastic deformation, but the greater stiffness and more sophisticated deformation relative to the articulated rotor hub, and the stronger flap-lag-torsion coupling motions, which lead to the issue of aeromechanical stability of a bearingless rotor helicopter is more complex and more prominent compared with the articulate and spherical flexible rotor hub of helicopter. The ground resonance and air resonance of a bearingless rotor helicopter are the key technology for the development models of helicopter, and a set of mature theory analysis method and effective experiment technology for the stability of a bearingless rotor on test bench is the basis of the bearingless rotor helicopter dynamic design.

In the aspects of the analysis approach and the experimental investigation of the effects of aeroelastic couplings on aeromechanical stability of a bearingless rotor helicopter, abroad have done a lot of analysis and test, have mastered the analysis and test method which verified by helicopter model, such as Boeing Apache and Sikorsky aircraft Comanche, have solved the bearingless rotor stability on technology 1-4. The study of aeromechanical stability of the bearingless rotor helicopter is relatively few in the domestic, Ling Aimin had built up a model of aeromechanic stability for a bearingless rotor helicopter by modal synthesis technique, had carried out the analysis of ground and air resonance, had studied the influence of some factors on stability, as reported in Ref.5. But in the respect of experimental technology of bearingless rotor and fuselageltest bench coupling dynamic stability, yet research in the domestic.

${ }^{a}$ Corresponding author: everyanyan@126.com 
For the case of a conventional ground and air resonance stability test, a rotor is mounted on a fuselage, and the test is within the nominal rotor speed, it is not only less test status but also more risky6. The present experiment method of ground and air resonance for a bearingless rotor on test bench is after theoretical analysis of ground and air resonance, and the results of the computation indicate there is no instability phenomenon within the range of test speeds, the stability margin of damping are adequate for the full range of resonance speeds. Only if these conditions are fulfilled can the stability test to carry out. An experimental program is undertaken with a relatively simple, smallscale model rotor and test bench to examine helicopter aeromechanical instabilities in a manner analogous to the theoretical approach of Ref.5. Initial experiments investigated the problems involves in making measurements of this nature, and obtains data for use in validating the theoretical model.

The paper starts with an introduction of test plan, the tested rotor model is descripted, test procedures are discussed, as well as the modal behavior is identified, and mode's frequency and damping are determined. A very brief discussion of the theoretical model of Ref. 8 is given, and then a comparison of analytical and experimental results is presented.

\section{Test scheme}

In order to validate the modeling analysis method of ground and air resonance of a bearingless rotor helicopter, and to provide a reliable test technique for the stability test, a bearingless model rotor of three blades have been designed and manufactured. Based on the hub center modal characteristics of the dynamic test bench, a test scheme could be developed according to the calculation results of resonance speed region and damping stability margins of ground and air resonance. Test scheme mainly includes the selection of test conditions, the excitation method, the measure methods and items, and the safety of the test bench and rotor system, the feasibility of test program, the comprehensive effectiveness of test results should be considered at the same time.

The specific test scheme as follows:

1) Select test conditions according to the ground resonance speed region of model analysis. If the coupling mode is stabilizing for the full range of resonance speeds, then select tree to five test speeds from low speed to high speed within resonance speed region. Otherwise, first select two speeds in the resonance speed region on the left side of the low speed stability region, and then accelerate over the speed instability region, afterwards select two speed in the resonance speed area on the right side of the high speed stability region, so that can begin to ground resonance test.

2) Use the frequency scanning sine excitation method, implement rotor blade longitudinal and transverse cycle pitch incentive by programming, the scanning frequency corresponding to a determine speed which is from low to high containing expected natural frequency of lead-lag.

3) Adopt the method of monitoring the flexbeam profile bending load and test-bed vibration level, control the excitation amplitude of the cycle pitch from small to large, control the excitation phase from short to long, always ensure the safety of the rotor system and test bench.

4) By changing the collective pitch to investigate the effect of rotor lift on stability of ground and air resonance.

5) Ground resonance test is in the state of vertical orientation of balance lock, air resonance test is in the state of vertical orientation of balance free.

\section{Experimental model}

The rotor model used in the experiment has a 4-m diameter, three-blade rotor which is mounted on a dynamic test bench. An overall view of the model is shown in Fig.1. The rotor was designed so that most of the blade flexibility is concentrated in root flexures. The bearingless rotor using three arms structure with soft in rotary plane. The core part is the composite flexi beam, blade flapping and leadlag and pitching motions is presented by the deformation of flexbeam. The variable pitch moment of blade root is passed on to the blade through the blade root sleeves. Blades adopt the composite structure, are mainly composed of girder and skin and foam filled inside. The pitch control forces of 
the rotor are from the swashplate, through the pitch rod to pitch horn, to blades, the pitch control of blades is implemented eventually. An overall view of one rotor is shown in Fig.2.

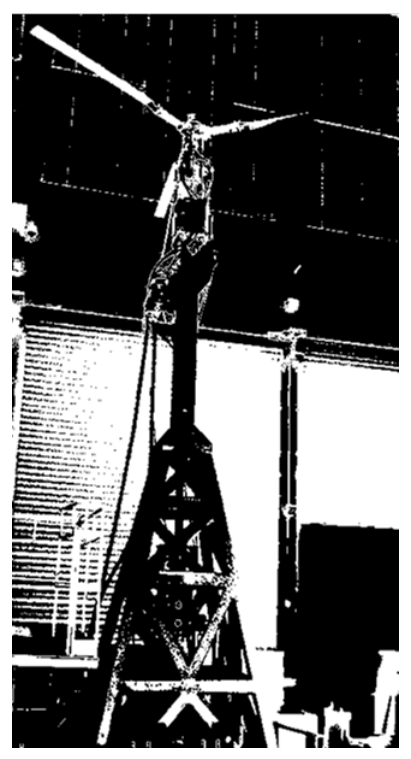

Figure 1. Overall view of model.

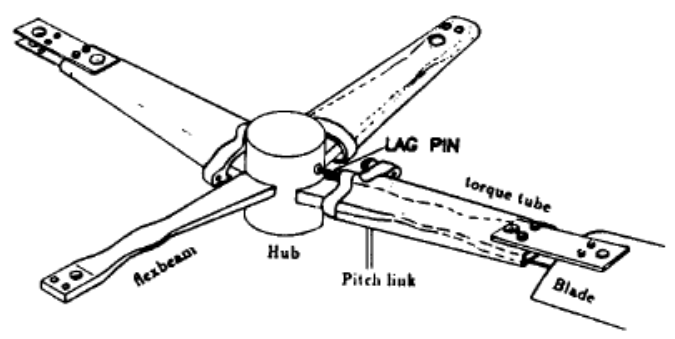

Figure 2. Configuration view of a bearingless rotor.

The test bench used in the experiment is the rotor dynamic response / aeroelastic stability test-bed in china helicopter research and development institute (referred to dynamic test-bed). The test-bed is bolted to the transmission and electric drive motor, power cables and cooling water for the motor, oil lines, thermocouples, and instrumentation, which together constitute the body of the model. The test bench is supported on a stand of considerable rigidity (see inset of Fig.1).

\section{Test procedures and data analysis}

The premise condition of ground and air resonance test is the calculation results shown that no instability in the range of test speeds, and have sufficient damping stability margin, after the complete calculation and analysis of ground and air resonance of a bearingless model rotor on dynamic test-bed.

Two acceleration sensors are attached to the hub center, are used to measure the acceleration of rotating surface in both $\mathrm{X}$ and $\mathrm{Y}$ directions. Three acceleration sensors are attached to the upper of test bench, are used to measure the acceleration of these points, meanwhile provide overload monitoring. Flap-lag strain gauge are stuck on opposite sides of the four sections of the flexbeam, to measure flapping and lead-lag bending stress of the flexbeam, and the strain gauge are full-bridge. 
The cycle of pitch incentive is implemented by controlling the swashplate.Using hydraulic exciting method, through shock up actuator cylinder, then incentive the non-rotating ring of swashplate, in order to realize the cycle of pitch incentive, and the excitation amplitude values remain constant. Collect the flap-lag-pitch time domain data of flexbeam and blade different sections in the area of before and after exciting, and the vibration data of body corresponding test status at the same time. A block diagram of test principle is shown in Fig.3.

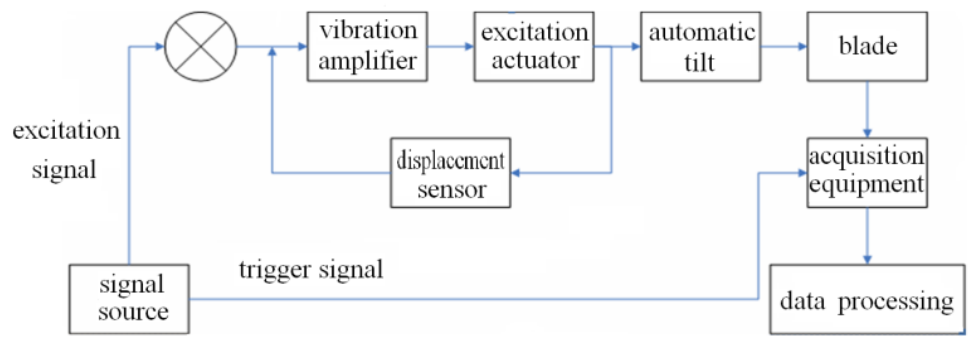

Figure 3. Block diagram of test principle.

Rotor speed was used as the primary variable in the experiment. At each rotor speed, the lead-lag regressing mode was excited, and normally two transient records were obtained, the data edited if necessary and the Fast Fourier Transform (FFT) of the signal obtained. The modal frequency identified, and the moving-block analysis was then used to identify the modal damping, the stability margin of ground and air resonances were calculated. In addition, the amplitude and phase of the mode in each coordinate was calculated, assisting in the identification of the modal behavior.

\section{Theoretical model}

The theoretical model used for comparison with the experimental results. The dynamic characteristic of rotor and blades were calculated using finite element model. The flexbeam, sleeve, blade, damper and pitch horn were simplified into a finite number of units, the relationship between the individual structure elements were dealt with the load path. The finite element model of a blade is described in Fig.4. There are six degrees of freedom with each node [u, v, w, $\theta$, w', v']. The dynamic characteristic of an isolated blade could be calculated, then the low modes of coupling modal were selected, they are the first lead-lag mode and the first flapping mode, and the corresponding mode-shape including the torsional component.

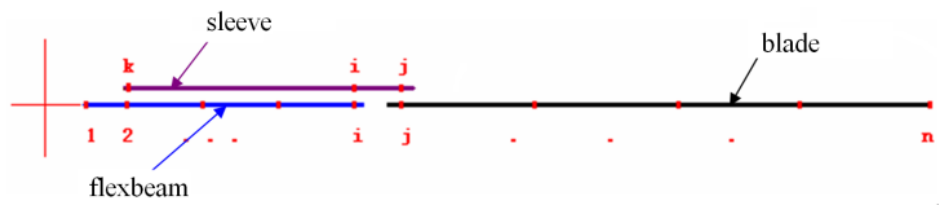

Figure 4. View of finite element model of a blade.

In the state of pitch and roll and vertical directions of control mechanism on dynamic test-bed are locked, the amplitude-frequency characteristic and phase-frequency characteristic of dynamic testbed's lateral-directional and side-directional were measured, and effective mass and stiffness and damping and frequency of hub center were measured too.

\section{Experimental results and comparison with theory lead-lag regressing mode damping for coupling}

The lead-lag regressing mode is the critical mode, as this is the mode that will become unstable underground or air resonance conditions. When a complete understanding of coupled rotor-body 
stability, this mode is especially true in comparing theory and experiment, and in assessing any limitations of the theoretical model. For all tests, the range of rotor speeds tested was included values from 850 to $1032 \mathrm{rpm}$. The damping and frequency of all the modes were tracked at the $0^{\circ}$ to $3^{\circ}$ collective pitch conditions. The modal damping measurements are compared with theory in Figs. 5 and 6 for the lead-lag regressive, at three rotor speeds within ground resonance speed region, and two rotor speeds within air resonance speed region.
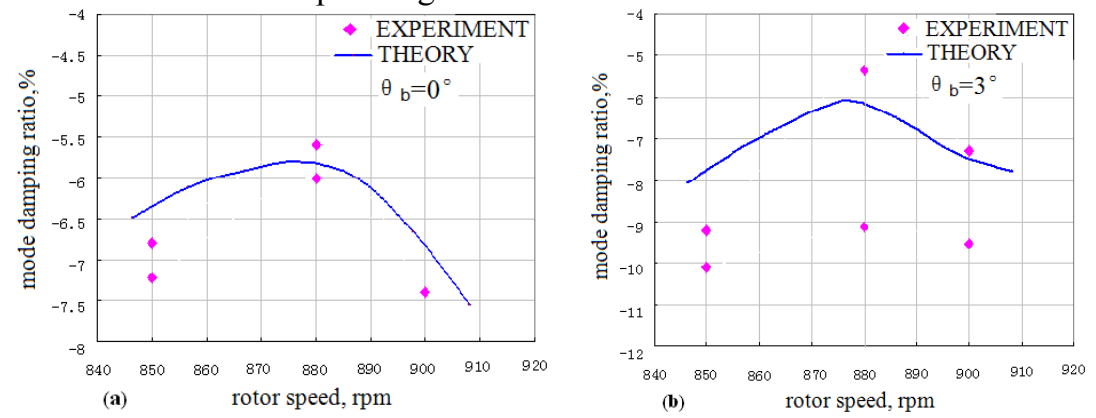

Figure 5. Lead-lag regressive mode damping as a function of rotor speed for ground resonance, in fixed coordinate, (a) $\theta_{\mathrm{b}}=0^{\circ}$, (b) $\theta_{\mathrm{b}}=3^{\circ}$.
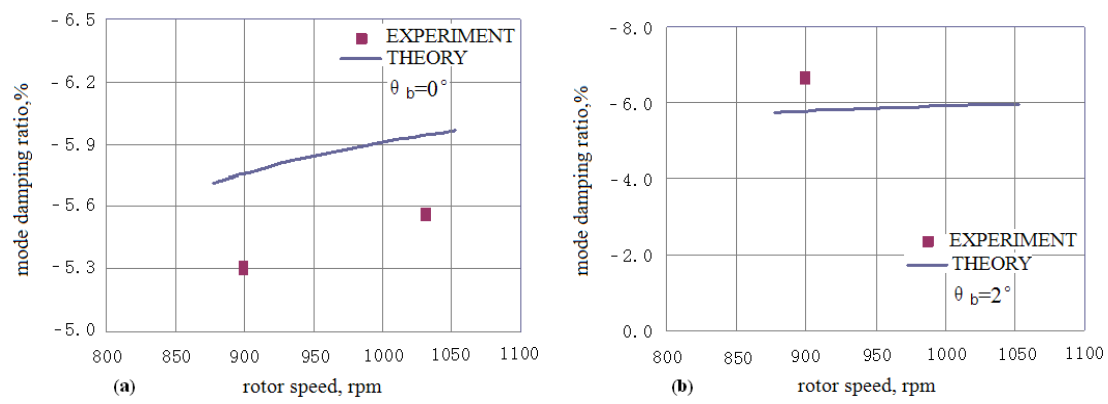

Figure 6. Lead-lag regressive mode damping as a function of rotor speed for air resonance, in fixed coordinate, (a) $\theta_{\mathrm{b}}=0^{\circ}$, (b) $\theta_{\mathrm{b}}=2^{\circ}$.

Fig.5, which represents the ground resonance case, as rotor speed increased there is a slight reduction in the damping as the lead-lag regressing mode couples with the body mode. Then, with a further increase in rotor speed, the damping is rising. Fig.6, which represents the air resonance case, the damping is rising as rotor speed increased. By definition the damping is stability over the full range of rotor speeds, the lead-lag regressing mode is stabilized and no instability occurs. The agreement of the experiment and theory is quite good, all deviation is less than $15 \%$. A partial objective of this effort is the value of the body structural damping.

Some conclusions have been obtained by comparing and analyzing the ground resonance and air resonance test result with the calculated result of a bearingless model rotor on dynamic test-bed.

(1) Correctness of the test incentive method, the concerned lead-lag regressing mode was excited, for all test status.

(2) For ground resonance and air resonance, within the nominal rotor speed $(\mathrm{n} \leq 1032 \mathrm{r} / \mathrm{min})$, as some variation in the collective pitch and excitation frequency, no unstable region, is steady over all rotor speed, no ground resonance and no air resonance occur.

(3) Damping stability margin were greater than $5.0 \%$ for ground resonance, more than $4.0 \%$ for air resonance.

(4) Compared the attenuation mode damping of ground and air resonance test results with the calculated results, the deviation is less than $15 \%$, the correlation between the experiment and the theory is quite good.

(5) Correctness of ground and air resonance experiment method of a bearingless model rotor on dynamic test-bed and theoretical calculation method is verified. 


\section{Conclusions}

In view of the new configuration of bearingless rotor, a test method of ground and air resonance in the resonance speed region was proposed in this paper, the test principle was described. The stability test of rotor model scale on test-bed was completed for the first time in domestic, and information about modal frequencies and damping was obtained. The experimental results were compared with theory for the lead-lag regressing mode damping, the correlation was generally quite good, and all the error value is less than $15 \%$. The reliability and effectiveness of the test and analysis method were verified, that reduced the risk of the traditional experiment, achieved the aim of experiment verified the stability margin. A set of ground resonance and air resonance test method for a bearingless rotor on test-bed and data processing method were formed, the test technique did suggest promising test approaches for a helicopter application.

\section{Acknowledgments}

This work is supported by the Fund of Aeronautics Science and National 863 Plan of China.

\section{References}

1. William G. Bousman. An Experimental Investigation of the Effects of Aeroelastic Couplings on Aeromechanical Stability of a Hingeless Rotor Helicopter [J]. Journal of the American Helicopter, 36(4):46-54, 1981.

2. Hoges D H. Aeromechanical Stability of Helicopter with Bearingless Main Rotor[R]. NASA TM784S9,1978.

3. Hoges D H. An Aeromechanical Stability Analysis for Bearingless Rotor Helicopter[R]. Journal of American Helicopter Society, 1979, 24 (1):2-9.

4. Wang J M.Theoretical and Experimental Research in Aeroelastic Stability of Advanced Bearingless Rotor for Future Helicopters[C]// 29th Aerospace Science Meeting.Reno Nevada,AIAA 1991-0192,1991.

5. Ai-Min Ling. Analysis of Aeromechanic Stability for Bearingless Rotor Helicopters. Journal of Nanjing University of Aeronautics \& Astronautics. 2011 43(3), in Chinese.

6. Fu-Hua Liu, Li Rui-Pin. Research and Application of Helicopter Ground Resonance Test Technologies. Technology \& Application. 2013.06 20(10), in Chinese. 